\title{
Gut microbiota in antiviral strategy from bats to humans: a missing link in COVID-19
}

\author{
Jia Luo ${ }^{1}$, Shan Liang ${ }^{2} \&$ Feng Jin ${ }^{3 *}$ \\ ${ }^{1}$ Department of Psychology, Sichuan Normal University, Chengdu 610068, China; \\ ${ }^{2}$ Key Laboratory of Microbial Physiological and Metabolic Engineering, Institute of Microbiology, Chinese Academy of Sciences, \\ Beijing 100101, China; \\ ${ }^{3}$ Key Laboratory of Mental Health, Institute of Psychology, Chinese Academy of Sciences, Beijing 100101, China
}

Received July 28, 2020; accepted September 21, 2020; published online January 27, 2021

\begin{abstract}
Bats are a potential natural reservoir for SARS-CoV-2 virus and other viruses detrimental to humans. Accumulated evidence has shown that, in their adaptation to a flight-based lifestyle, remodeling of the gut microbiota in bats may have contributed to immune tolerance to viruses. This evidence from bats provides profound insights into the potential influence of gut microbiota in COVID-19 disease in humans. Here, we highlight recent advances in our understanding of the mechanisms by which the gut microbiota helps bats tolerate deadly viruses, and summarize the current clinical evidence on the influence of gut microbiota on the susceptibility to SARS-CoV-2 infection and risk of COVID-19 leading to a fatal outcome. In addition, we discuss the implications of gut microbiota-targeted approaches for preventing infection and reducing disease severity in COVID-19 patients.
\end{abstract}

COVID-19, SARS-CoV-2, gut microbiota, bat, anti-inflammatory, antiviral

Citation: Luo, J., Liang, S., and Jin, F. (2021). Gut microbiota in antiviral strategy from bats to humans: a missing link in COVID-19. Sci China Life Sci 64, 942-956. https://doi.org/10.1007/s11427-020-1847-7

\section{Introduction}

The outbreak of coronavirus disease 2019 (COVID-19) has been declared a public health emergency of international concern by the World Health Organization. Currently, in the face of the rapidly spreading disease and a large number of infected people, there is an urgent need for effective measures to prevent infection and avoid fatal outcomes in COVID-19. The more we learn about the novel SARS-CoV2 virus and its associated outbreak, the better we can respond. In this context, understanding the interaction of SARS-CoV-2 with its natural host may be useful. However, the origin of SARS-CoV-2 remains unclear, although bats are currently implicated (Andersen et al., 2020; Zhou et al., 2020a; Zhou et al., 2020b). Bats are a natural reservoir for

*Corresponding author (email: jinfeng@psych.ac.cn) various viruses and noted for their ability to co-exist with some of the world's deadliest viruses, such as MERS-CoV, SARS-CoV, Nipah, Hendra, and Ebola viruses (Li et al., 2020c; Wang and Anderson, 2019). Via such co-existence, viral infections that are highly aggressive in humans can be persistent and avirulent in bats. Exploring the mechanism by which bats tolerate viruses can help us understand why humans are susceptible to SARS-CoV-2 and how we can coexist with them while staying healthy, or at the very least reduce the disease severity.

Compared with humans and other mammals, bats have two unique features: tolerance to viruses and an ability to fly. What if the presence of one condition influenced the likelihood of developing the other? Previous genomic insights hinted at this connection because, via whole-genome sequencing of wild-caught bats, it was found that genes involved in minimizing DNA damage as a metabolic 
consequence of flight have been linked to immune function (Zhang et al., 2013). The increased rate of metabolism accompanying flight would lead to higher levels of oxygenfree radicals. This makes bats more prone to generating damaged DNA. Such excessive inflammation is energetically expensive and is detrimental, potentially having pathological consequences. Therefore, bats have evolved mechanisms to suppress the activation of immune responses due to damaged DNA generated by the energy used for flight, leading to reduced inflammation (Subudhi et al., 2019). Results from cell culture experiments in bats have shown that inflammation was suppressed by dampening the activation of DNA sensors, such as stimulator of interferon genes (Xie et al., 2018), and reducing levels of inflammatory cytokines, such as tumor necrosis factor alpha (TNF- $\alpha$ ) (Banerjee et al., 2017). However, the suppression of inflammation would consequently induce greater susceptibility to virus infection. The evolutionary suppression of inflammation is counteracted by the constitutive expression of innate immune genes or novel genes targeting viruses in bats, such as higher constitutive expression of interferon and interferon-stimulated genes (ISGs) (Banerjee et al., 2020). Thus, genomic insights have generated this plausible explanation for how bats may have evolved to be able to harbor such deadly viruses without experiencing disease.

In addition to the host genome, abundant evidence has shown that the microbiome in the gut directly regulates the host immune response to viral infection (Honda and Littman, 2016; Thaiss et al., 2016). However, no integrative study has yet been performed addressing whether gut microbiota contributes to bats' innate ability to act as a viral reservoir. Recently, it has been revealed that bats have a unique gut microbiota, in terms of its diversity and composition, as a consequence of physiological adaptations to flight. Is there a potential relationship among the three unique features of bats? Is it possible that the gut microbiota is the missing link between adaptation to flight and tolerance of viruses? By what mechanism does the gut microbiota help to harbor viruses, and is this pattern consistent in humans? We believe that studies of gut microbiota in bats can help to answer these important questions and more. More crucially, understanding the role of the gut microbiota in bats controlling virusmediated pathogenesis may enable researchers to identify novel therapeutic targets to prevent and treat infections with SARS-CoV-2 in humans.

\section{Gut microbiota: a missing link between flight and immunity in bats}

\section{Adaptation to flight structuring bats' gut microbiota}

As a form of co-evolution, the adaptation of mammals to their diverse lifestyles has played a key role in structuring their gut microbiota (Rothschild et al., 2018). In bats, the adaptation to powered flight placed selective pressures on the assembly of gut microbiota. The pressure to decrease mass for more efficient powered flight reduces the microbial biomass in the gut, as it has been shown that bats carry much lower bacterial loads in the feces than nonflying mammals (Song et al., 2020a). In addition, as a by-product of selective pressure to decrease mass, bats have reduced their intestinal lengths and shortened intestinal content retention times (Caviedes-Vidal et al., 2007). Shorter guts, with correspondingly reduced anaerobic volumes, could present less of a barrier to microbial exchange through the aerobic environment. Thus, gut microbial reduction is common in bats, displaying markedly less gut microbial richness and diversity than nonflying mammals. Consistently lower resident gut microbiota might increase the proportion of transient environmental microbes relative to the total gut microbiota (Song et al., 2020a). As a result, the bats' gut microbiota consists of bacteria from their environment rather than from an evolutionary predisposition to host-specific bacteria. This is supported by evidence that the geographic locality is a strong predictor of gut microbiota composition in bats. Indeed, it has been shown that the same species of bat in five geographic localities might host five very different gut microbial communities (Lutz et al., 2019).

The discovery that adaptation to flight leads the gut microbiota of bats to be closely linked to the environment around them may suggest that bats are more highly tolerant of viruses than other mammals. In other mammals, maintaining a stable gut microbiota is achieved by eliminating invasive pathogens. However, the possession of a transient environmental gut microbiota by bats may have led to the evolution of a quite different antiviral strategy (Figure 1).

\section{Gut microbiota in bats is linked to their unique immune responses to viruses}

Bats harbor a significantly different gut microbiota composition compared with other mammals. At the phylum level, Proteobacteria overwhelmingly dominates bats' gut, followed by Firmicutes and Tenericutes, along with a few Bacteroidetes across plant- and animal-feeding bats. At the family level, the Enterobacteriaceae species in the phylum Proteobacteria are the most prevalent bacteria ( $\mathrm{Li}$ et al., 2018). Interestingly, in humans, enrichment of Proteobacteria is a microbial signature of dysbiosis in gut microbiota, and Enterobacteriaceae is considered to be a potential pathogen (Shin et al., 2015).

Another unique microbial signature of bats is the amount of Bacteroidetes, which is significantly low in bats, but is one of the most abundant bacterial phyla in other mammals (Li et al., 2018). It is widely accepted that the gut microbial signature of high Proteobacteria and low Bacteroidetes does not 


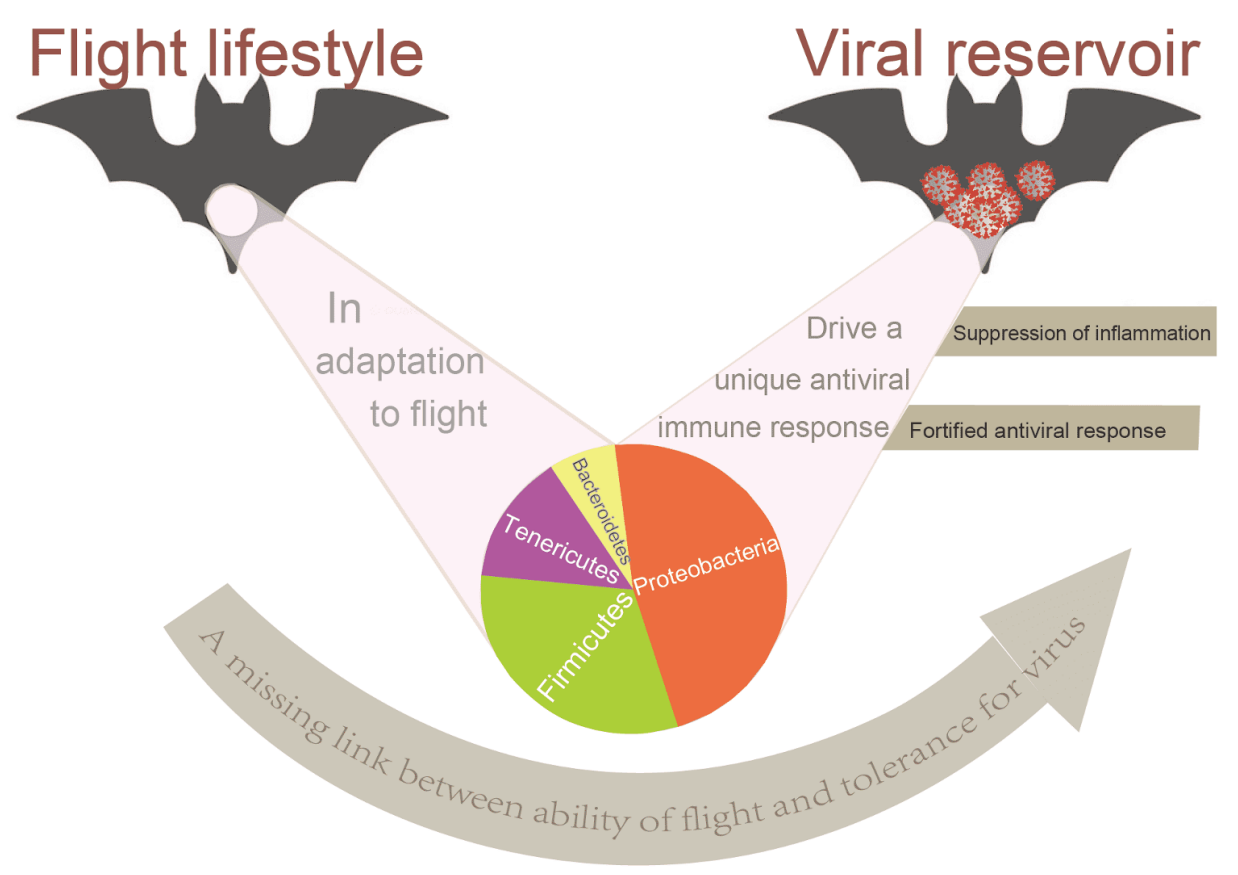

Figure 1 (Color online) Gut microbiota: a missing link between ability of flight and tolerance for virus in bats. The adaptation to powered flight of bats exerts selective pressures on the assembly of gut microbiota. The pressure to decrease mass for more efficient powered flight reduced the gut microbial biomass, displaying markedly less gut microbial richness and diversity than most nonflying mammals. Consistently lower resident microbial biomass might increase the proportion of transient environmental microbes relative to the total pool of microbes in gut. As a result, the bats' gut microbiota consist of bacteria that the bats pick up from their environment rather than from an evolutionary predisposition to host-specific bacteria, harboring a significantly different gut microbiota composition compared to other mammals. In particular, Proteobacteria overwhelmingly dominated bat guts, and was followed by Firmicutes and Tenericutes, along with a few Bacteroidetes. It has been shown that centenarians and Hadza hunter-gathers, who have a higher tolerance for Proteobacteria, have better immune resistance to inflammatory and infectious diseases. So, co-existing with a gut microbiota characterized by high Proteobacteria and low Bacteroidetes may set the basal status of immune response to virus in bats to a "tolerance status" with suppression of inflammation and fortified antiviral response, contributing to bats' innate ability to act as viral reservoir. Evidence from accumulated studies of gut microbial modifications (the germ-free animals, pathogen infection, antibiotic treatment and fecal microbiota transplantation) in bats and other mammals may support this notion.

meet the established standard parameters of a "healthy microbiota" in humans (Rinninella et al., 2019). Not only that, the gut microbial signature of bats is associated with various inflammatory diseases in humans. In both Crohn's disease and ulcerative colitis patients, the gut microbial diversity is significantly reduced, along with an increase in the phylum Proteobacteria, enrichment of the family Enterobacteriaceae, and depletion in the phylum Bacteroidetes (Eom et al., 2018; Franzosa et al., 2019). In ankylosing spondylitis patients, who often have subclinical gut inflammation or clinically defined inflammatory bowel disease (IBD), the gut microbiota is characterized by markedly decreased diversity, increased abundance of Proteobacteria, and decreased Bacteroidetes ( $\mathrm{Li}$ et al., 2019). In addition, in cystic fibrosis patients who usually suffer from chronic gut inflammation, the predominant phylum is Proteobacteria (mainly Enterobacteriales), followed by Firmicutes and finally a few Bacteroidetes. Furthermore, probiotic intake not only significantly reduces Proteobacteria and increases Bacteroidetes, but also markedly improves gut inflammation (del Campo et al., 2014).

Interestingly, proliferation of undesirable Proteobacteria at the cost of symbiont Bacteroidetes was also found in cen- tenarians, when compared with the young and elderly (Santoro et al., 2018). In a co-evolutionary vision of the relationship between gut microbiota and the host, centenarians who achieve healthy longevity might be those whose gut microbiota manages to continuously re-establish a mutualistic relationship with the host, adapting to the progressive endogenous and environmental changes (Biagi et al., 2017). This age-related remodeling of gut microbiota may subsequently contribute to shaping the immune status in centenarians. Previous publications have asserted that inflammation, a chronic low-grade inflammatory status, is a typical characteristic of the elderly. However, centenarians are unique in that they not only have a lower propensity to mount inflammatory responses but also exhibit efficient antiinflammatory networks that may reduce disease severity. Therefore, the gut microbiota-driven decrease of chronic inflammation without compromising excessive inflammatory response when exposed to pathogens is the key to healthy aging and longevity (Franceschi et al., 2007).

It seems that centenarians tolerate the consolidation of a new gut microbiota ecosystem balance, resembling a property typical of the bats' gut microbiota composition. Accordingly, it is reasonable to speculate that, in adaptation to 
the flight-related progressive endogenous and environmental changes, remodeling of the gut microbiota in bats may contribute to immune tolerance to viruses (Figure 1). In support of this notion, compared with Westernized populations, the Hadza hunter-gatherers, who have higher tolerance for pathogenic symbiont gut microbiota, such as members of Proteobacteria, Succinivibrio, and Treponema, are less susceptible to inflammatory and infectious diseases (Rampelli et al., 2015; Schnorr et al., 2014).

\section{Gut microbiota driving the antiviral strategy in bats}

Co-existence with a unique gut microbiota may set the basal status of immune responses to viruses to a "tolerant" status, contributing to bats' innate ability to act as viral pathogen reservoirs. One of the unique immune responses to viruses in bats is the suppression of excessive virus-induced inflammation, which has been consistently attributed to reduced expression of inflammatory cytokines, such as IL-1 $\beta$ and TNF- $\alpha$ (Banerjee et al., 2020; Subudhi et al., 2019). The ability to suppress the pro-inflammatory cytokines TNF- $\alpha$ and IL- $1 \beta$ is directly regulated by gut microbiota, which has been shown in accumulated studies of gut microbial modifications including in germ-free animals, pathogen infection, antibiotic treatment, and fecal microbiota transplantation (FMT) (Lin et al., 2019; Rosser et al., 2014; Sun et al., 2018). Additionally, the low levels of NOD-like receptor protein 3 (NLRP3) inflammasome activation in bats have been found to account for the suppression of excessive virus-induced inflammation (Ahn et al., 2019). However, the extent to which NLRP3 signaling is activated appears to be greatly influenced by the composition and function of the gut microbiota (Seo et al., 2015; Singh et al., 2019). To act as a viral reservoir, the defect of suppressed inflammation is counteracted by a more efficient antiviral defense system to target viruses in bats. Studies have shown that interferon (IFN) and interferon-stimulated genes (ISGs) were constitutively expressed at higher levels in bats than in other mammals (Hölzer et al., 2019; Zhou et al., 2016), which suppressed viruses down to levels of persistence. The gut microbiota is considered to be involved in regulating bats' fortified antiviral defenses (Figure 1). Results from antibiotic treatment and fecal transplantation demonstrated that the gut microbiota can act at multiple levels to enhance antiviral resistance. This process includes increasing IFN production and ISG induction in the lungs to control viral infection early on, and enhancing the functionality of immune cells leading to improved innate and adaptive immunity later in viral infection (Bradley et al., 2019; Steed et al., 2017).

Although this is indirect evidence, studies of gut microbial modifications in bats would provide further support for the role of gut microbiota in bats' unique antiviral responses. Previous studies in bats showed that diet and pathogenic infection induced changes in gut microbiota composition in a way that could have an impact on immune responses to viruses. Consuming blood is an extreme evolutionary specialization, as blood is a nutrient-poor resource that is low in carbohydrates and vitamins. The common vampire bat (Desmodus rotundus) is one of only three mammalian species that feed exclusively on blood. Evidence has shown that this dietary specialization may cause vampire bats to have a gut microbiota harboring more pathogenic bacterial species, contributing to a more efficient immune system for antiviral defense (Zepeda Mendoza et al., 2018). Another study further elucidated how dietary changes in Desmodus rotundus influenced its immune response to viral infection. Results revealed that, compared with feeding on blood of wild animals in a forest fragment, bats feeding on blood of livestock showed changes in the relative abundance of several core bacterial taxa, which may decrease immune resistance to viral infection (Ingala et al., 2019). In a pathogenic infection model, it has been revealed that infection with astrovirus induced a higher pathobiont-like shift in the gut microbiota of young compared with adult bats. This different change in gut microbiota composition between young and adult bats was suggested to be responsible for their distinct immune responses to infection, as adult bats have a better immune defense against astrovirus infection and other enteric pathogens, whereas young bats often develop an increased severity of disease after infection (Wasimuddin et al., 2018).

\section{Findings from bats: implications for COVID-19 crisis}

Hosts and their own unique gut microbiota achieve co-existence over a long history of mutualistic symbiosis, in which gut microbiota has the potential to promote the host's health and provide defenses against invading pathogens. What we learn from bats is that harboring gut microbiota with a microbial signature of dysbiosis in humans is responsible for the suppression of inflammation and fortified antiviral responses, contributing to deadly virus defense and reduction of disease severity. This information from the natural host of SARS-CoV-2 highlights the potential importance of gut microbiota in SARS-CoV-2 infection and disease progression in humans. As such, the gut microbiota can be explored for potential natural defenses against a fatal outcome of COVID-19 in humans.

\section{Involvement of gut microbiota in COVID-19}

The COVID-19 pandemic has rapidly spread all over the world. The virus most likely infects respiratory epithelial cells and spreads mainly via the respiratory tract from human to human, as the viral infection causes a series of respiratory 
illnesses including severe respiratory syndrome (Mu et al., 2020). However, recent publications have suggested that the value of intestinal immunity and gut microbiota may be underestimated in COVID-19. In support of this, clinical observations showed that the viral receptor angiotensinconverting enzyme 2 (ACE2) used by SARS-CoV-2 for the entry process is highly expressed in the gut and linked to intestinal inflammation (Hashimoto et al., 2012; Letko et al., 2020; Xiao et al., 2020), and approximately $10 \%$ of patients with SARS-CoV-2 infection present diarrhea (Song et al., 2020b). In addition, COVID-19 patients showed gut microbial dysbiosis with reduced diversity, a significantly increased relative abundance of opportunistic pathogens, and a decreased abundance of beneficial symbionts, such as Lactobacillus and Bifidobacterium (Silan Gu et al., 2020; Xu K et al., 2020; Zuo et al., 2020c). Furthermore, serial studies recently provided convincing evidence supporting the involvement of the gut microbiota in intestinal SARS-CoV-2 infection, as it was reported that SARS-CoV-2 RNA was detected in stool samples (Yeo et al., 2020), active SARSCoV-2 replication was observed in human intestinal epithelial cells and COVID-19 patients' intestinal tissue (Qian et al., 2020; Stanifer et al., 2020), as well as SARS-CoV-2 intestinal viral activity being associated with gut microbiota composition in COVID-19 patients (Zuo et al., 2020a). These publications may suggest that there is interplay between intestinal SARS-CoV-2 and the gut microbiota in COVID-19 patients. Evidence has shown that a lack of ACE2 led to obvious alteration in the gut microbiota composition in mice, which resulted from reduced production of the antimicrobial peptides that control the gut microbial community (Hashimoto et al., 2012). Moreover, loss of ACE2 during pulmonary hypertension is associated with gut microbiota dysbiosis in humans (Kim et al., 2020). It seems that SARS-CoV-2 infection induced reduction of ACE2 can lead to alteration in the composition of the gut microbiota. In parallel with this, alterations in gut microbiota may influence the intestinal SARS-CoV-2 activity. Recent studies have reported that Bacteroides species, which downregulated ACE2 expression in murine gut, correlated inversely with intestinal SARS-CoV-2 load in COVID-19 patients (Zuo et al., 2020c). However, there is a need for further studies to determine the role of the gut microbiota in intestinal SARS-CoV-2 activity, which should be investigated in relevant animal models of COVID-19 by performing fecal transfer experiments. Possible models include non-human primates, ferrets, humanACE2-expressing mice, and hamsters (Callaway, 2020).

Following intestinal SARS-CoV-2 infection, the altered gut microbiota may then regulate the gastrointestinal symptoms in COVID-19 patients. It has been shown that the ACE2-mediated intestinal inflammation was greatly influenced by the composition and function of the gut microbiota, as transplantation of the altered gut microbiota from Ace2 mutant mice into germ-free wild-type hosts was able to transmit a highly increased propensity to develop severe colitis (Hashimoto et al., 2012).

Intriguingly, compared with a profile showing high expression of ACE2 in the human gut, the expression of ACE2 was quite low in lung tissues from healthy donors (Liang et al., 2020). This significant difference has raised concerns about the pathological importance of gut microbiota in lung infection with SARS-CoV-2 and the subsequent severe respiratory syndrome. Indeed, a recent report demonstrated that alterations in gut microbiota of patients with SARSCoV-2 infection were associated with the severity of COVID-19 respiratory disease (Zuo et al., 2020c). However, how can microbiota in the gut communicate with the lungs from a distance? Emerging experimental and epidemiological evidence highlights crucial cross-talk between the gut microbiota and the lungs, termed the gut-lung axis, which includes several pathways (Figure 2). (1) The mesenteric lymphatic system pathway is an essential pathway between the gut and the lungs, through which the gut microbiota-derived components and metabolites, such as short-chain fatty acids (SCFAs), may translocate across the intestinal barrier, reach the systemic circulation, and modulate the lung immune response (McAleer and Kolls, 2018). (2) While SCFAs have direct effects on the lungs, they also alter lung health through changes in bone marrow hematopoiesis. Many innate and adaptive immune cells that populate the lungs originate in the bone marrow. Thus, SCFAs can shape and promote bone marrow hematopoiesis to resolve pulmonary inflammation and to support healthy homeostasis (Trompette et al., 2014). (3) The gut is also a source of immune cells for the lungs. The innate lymphoid cells that are involved in tissue repair have been shown to be recruited from the gut to the lungs in response to inflammatory signals in the presence of IL-25 (Huang et al., 2018). (4) Gut microbiota can enhance IFN production and ISG induction in the lungs to increase antiviral resistance to viral infection (Bradley et al., 2019). (5) Gut microbiota can influence pulmonary microbial composition by both direct seeding of the respiratory tract with bacteria and the distribution of bacterial metabolites, such as SCFAs, which promote the growth of certain SCFA-producing bacteria (Marsland et al., 2015).

Clinical reports have shown that COVID-19 affects not only the respiratory and gastrointestinal systems, but also the central nervous system. Indeed, neurological manifestations related to COVID-19 have been addressed in large studies. The specific neurological symptoms in COVID-19 patients normally include acute cerebrovascular disease, ataxia, seizure, cranial nerve involvement, and neuroinflammationassociated neurological disorders (Asadi-Pooya and Simani, 2020; Beyrouti et al., 2020; Kempuraj et al., 2020). In addition to direct CNS invasion, microthrombosis, severe hypoxemia, and neurological side effects of antiviral 


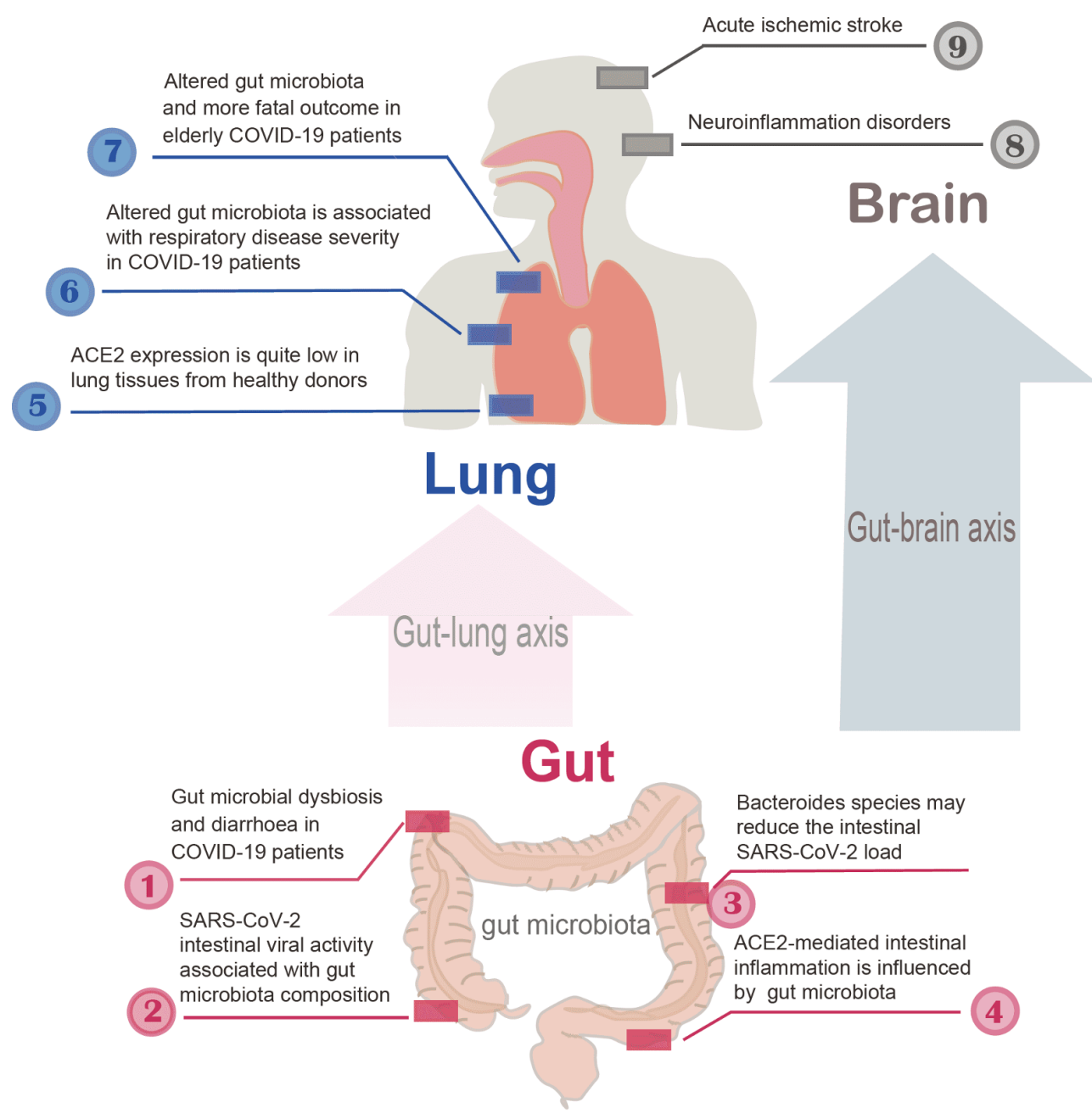

Figure 2 (Color online) The potential influence of gut microbiota on COVID-19 disease outcome. Gut micorbiota is associated with intestinal SARS-CoV2 infection and plays a vital role in gastrointestinal symptom (1)-(4) of COVID-19. Subsequently, the influence of gut microbiota reaches the lung and brain through the gut-lung axis and gut-brain axis respectively, contributing to respiratory disease severity (5)-(7)) and neurological manifestations in COVID-19 (8)-(9)).

medications, the mechanism of gut microbial dysbiosis has been proposed to explain the neurological damage produced by COVID-19 (Sultana and Ananthapur, 2020; Whittaker et al., 2020). Alteration of the composition of the gut microbiota during intestinal SARS-CoV-2 infection could be involved in the pathogenesis of neuropsychiatric symptoms via the gut-brain axis, including the immunological, hormonal, and neuronal pathways. A large number of studies have shown that the gut microbiota can affect various neurological symptoms and mental functions, such as anxiety, depression, multiple sclerosis, seizure, Parkinson's disease, autism, and Alzheimer's disease (Cryan et al., 2019).

There is now a growing body of evidence about how gut microbial dysbiosis negatively impacts neurological manifestations in COVID-19 patients. It has been reported that the neurological manifestations normally have a higher incidence in patients with a more severe course of COVID-19 (Chen et al., 2020). As reported in elderly and critically ill COVID-19 patients, their altered gut microbiota contributes to the IL-6-mediated cytokine storm (Thevaranjan et al., 2017), which influences the extent of acute respiratory distress syndrome, the pathogenesis of neuroinflammation, and other neurological disorders (Kempuraj et al., 2020; Muccioli et al., 2020). Therefore, the gut microbial dysbiosis in COVID-19 could modulate the neurological manifestations via the immunological pathway of the gut-brain axis. This is well described in acute ischemic stroke and neuroinflammatory disorders (Figure 2). In a recent paper, the gut microbiota has been described to regulate the onset of acute ischemic stroke through the immunological pathway (Battaglini et al., 2020). In respect to neuroinflammatory disorders, recent reports have revealed that COVID-19 might represent a risk factor for Parkinson's disease (PD). Previous studies have suggested that the pathogenesis of PD is strongly associated with gut microbial dysbiosis and colon inflammation. Indeed, several lines of evidence support the hypothesis that PD first begins in the gut and then spreads to the CNS (Sampson et al., 2016; Yang et al., 2019). As de- 
scribed earlier, significantly altered gut microbiota composition was observed in COVID-19 patients, and gut microbial dysbiosis was found to contribute to the IL-6-mediated cytokine storm. This increase in pro-inflammatory cytokines may then compromise intestinal barrier integrity, causing elevation of circulating lipopolysaccharides (LPS), which might eventually trigger microglial activation and neuroinflammation. Additionally, the gut microbial dysbiosis may modulate the symptoms of PD in COVID-19 patients via the neuronal pathway of the gut-brain axis. One histopathological hallmark of PD is the intraneuronal accumulation of aggregates of the protein $\alpha$-synuclein (aSyn). In this respect, gut microbial dysbiosis-mediated LPS elevation can stimulate the formation of aSyn in enteric nerves, which occurs prior to the onset of motor symptoms. In support of this, treatment with a specific gut bacterium that is markedly increased in PD mouse models was found to be sufficient to provoke the selective death of dopamine neurons and motor deficits in mice, accompanied by neuroinflammation and accumulation of aSyn in both colon and brain (Follmer, 2020). Although it is mechanistically feasible that the gut microbiota is involved in the pathogenesis of neuropsychiatric symptoms in COVID-19 infection via the gutbrain axis, the role of gut microbial dysbiosis in this setting remains to be investigated in clinical studies.

\section{The role of gut microbiota in increased risk for severe COVID-19}

The spread of COVID-19 throughout the world has been a severe challenge for public health. There is an urgent need to explore the essential reasons for the susceptibility to SARSCoV-2 infection and increased risk for a severe COVID-19 outcome. According to the important influence of the gut microbiota in COVID-19 infection and disease outcome, we propose that the disruption of gut microbial homeostasis may lead to an increased infection risk and poor outcome of COVID-19.

The gut microbial homeostasis of modern urban residents has been significantly perturbed by dramatic changes in the diet and lifestyle, increased antibiotic use, and excessive hygiene (Hills et al., 2019). For instance, urban residents typically consume a high-fat and high-refined-carbohydrate diet, which is rich in sucrose and fructose but lacks dietary fiber, perturbing the gut microbiota (Tomova et al., 2019). In addition, significantly reduced physical activity and disrupted circadian rhythm could also disrupt the gut microbiota (Matenchuk et al., 2020; O'Sullivan et al., 2015). In comparison, dwellers in remote rural areas who often have a traditional lifestyle maintain gut microbial homeostasis. It has been shown that, compared with rural African children in an environment that still resembles that of Neolithic subsistence farmers, Western European children have reduced gut microbial diversity and gut microbial dysbiosis associated with inflammatory diseases (De Filippo et al., 2010). This perturbed composition of the gut microbiota may contribute to the increased risk of respiratory tract infection as well as inflammatory diseases. Global comparisons revealed that lifestyle practices, such as caesarian section and antibiotic use, reduced the diversity and altered the composition of the gut microbiota, leading to a predisposition to asthma and an increased risk of respiratory virus infection and more severe disease outcomes (Shao et al., 2019).

It is noteworthy that people with more risk factors disrupting the homeostasis of the gut microbiota may have increased susceptibility to SARS-CoV-2 infection and risk of developing severe and fatal COVID-19. Thus, we propose that more attention should be paid to individuals with gut microbial dysbiosis during the COVID-19 outbreak. A special focus on this issue may help us better understand why some patients are completely asymptomatic, some develop mild symptoms, while others suffer acute respiratory distress syndrome. Accordingly, more insight into the pathological importance of the gut microbiota in the elderly should be obtained, since studies have shown that patients aged 65 years and older have an increased risk for SARS-CoV-2 infection and higher mortality of COVID-19 pneumonia (Du et al., 2020; Yuan et al., 2020). This increased risk in the elderly is not due to enriched ACE2 receptor expression, which is a prime target for the pathogenesis of COVID-19 (Li et al., 2020b). In fact, ACE2 expression has been shown to be dramatically reduced in the lungs of the elderly (AlGhatrif et al., 2020). However, compared with young adults, the elderly are known to have an altered composition of the gut microbiota. A number of studies have shown that a significant drop in diversity and decreased abundance of beneficial bacterial species are accompanied by the outgrowth of pathogenic species in the gut microbiota of the elderly. In terms of more specific details at the genus and family levels, Firmicutes and Actinobacteria (mainly Bifidobacteria) were found to be decreased, whereas Bacteroidetes and Proteobacteria (especially Enterobacteriaceae) were increased in the elderly (O'Toole PW and IB., 2015; Odamaki et al., 2016). Interestingly, the alteration in gut microbiota in the elderly is similar to the changes induced by antibiotic treatment, although both drug-related factors (such as antibiotic class, timing of exposure, or route of administration) and host-related factors (such as age at exposure) influence the alterations of gut microbiota produced by antibiotics. Based on results from different studies, antibiotic treatment was shown to result in a reduction in total bacterial diversity, and decreases of Firmicutes (mainly Lactobacilli) and Actinobacteria (especially Bifidobacteriaceae and Bifidobacteria), but an increase in Bacteroidetes and Proteobacteria (particularly Enterobacteriaceae) (Francino, 2016; Ianiro et al., 2016). 
As described earlier, antibiotic treatment that disrupts the gut microbiota results in significantly reduced immune responses against influenza virus and a worse outcome in pulmonary infection. Therefore, it is reasonable to speculate that the altered composition of the gut microbiota in the elderly may contribute to a higher risk for severe COVID-19 (Figure 2). Mechanistically, the cause of mortality in viral SARS-CoV-2 pneumonia is uncontrolled inflammation, whereby a large number of immune cells and substantial tissue fluid in the lung can block gas exchange between the alveoli and capillaries, leading to acute respiratory distress syndrome. It has been found that a rapid increase in the proinflammatory cytokine IL-6 plays a key role in the formation of this lethal cytokine storm (Chen and Zhong, 2020). According to animal and human studies, aged gut microbiota can cause higher IL-6 and peribronchiolar cellular infiltration in the lungs in the absence of stimulation or overt infection, increasing the risk for a fatal outcome of COVID-19 (Fransen et al., 2017; Thevaranjan et al., 2017).

These results suggest the potential value of the gut microbiota as a diagnostic biomarker for COVID-19. Indeed, several studies have shown that patients with chronic kidney disease, type 2 diabetes, hypertension, obesity, and non-alcoholic fatty liver disease, who had gut microbial dysbiosis, were at a higher risk of, and mortality from, COVID-19 infection (Bornstein et al., 2020; Fang et al., 2020; Finer et al., 2020; Henry and Lippi, 2020; Prins and Olinga, 2020). Although the increased risk in these conditions may be partly due to the increased ACE2 expression and treatment with ACE inhibitors, which result in upregulation of ACE2 (Fang et al., 2020), the specific composition of the gut microbiota linked to a severe COVID-19 outcome still needs to be addressed.

\section{Gut microbiota as a potential target against COVID-19}

On account of the importance of the gut microbiota in the susceptibility to SARS-CoV-2 infection and the risk of a fatal COVID-19 outcome, it is suggested that the gut microbiota may be able to act as a therapeutic target against COVID-19. What we know from bats is that the maintenance of gut microbial homeostasis with a high tolerance for pathogenic symbiont gut microbiota, such as members of Proteobacteria, may contribute to a marked reduction in disease severity of viral infection. This may suggest that the establishment of a mutualistic symbiotic relationship with Proteobacteria may avoid poor outcomes in COVID-19, as centenarians and Hadza hunter-gatherers, who have higher tolerance for Proteobacteria, have better immune resistance to inflammatory and infectious diseases. However, intolerance to members of the Proteobacteria may lead to various inflammatory diseases and an increased risk of viral infection in humans. In mammals, it has been widely recognized that exposure to specific microbes early in life plays an instrumental role in facilitating tolerance to the gut microbiota, which subsequently has long-lasting consequences for the immune response that extend into adulthood (Gensollen et al., 2016). Exposure to microbiota during early childhood contributes to the suppression of excessive inflammatory responses in adulthood, which is supported by the fact that early-life microbial exposure is associated with protection from inflammatory diseases such as IBD and asthma. In contrast, exposure to antibiotics in childhood, especially during the first year of life, was found to be associated with an increased risk for developing IBD, in which basal expression levels of the inflammatory cytokines were increased (Torres and Peter, 2019). Therefore, exposure to Proteobacteria early in life may facilitate the establishment of a mutualistic symbiotic relationship with Proteobacteria and subsequent reduction in disease severity upon viral infection. This may suggest that individuals growing up in Proteobacteria-rich environments would have a reduced risk of COVID-19. One of the environments dominated by Proteobacteria is a soil-rich natural environment, as frequently encountered by those in rural areas. This may provide an explanation for why urban residents growing up in environments with degraded soil have markedly less tolerance to Proteobacteria, a higher risk of inflammatory diseases, and increased risk of viral infection, compared with residents in rural areas (Tasnim et al., 2017). Besides a high tolerance for Proteobacteria, increased gut microbiota diversity and enriched abundance of beneficial bacterial species have also been shown to enhance the immune defense against viral infection in bats (Wasimuddin et al., 2018). Consistent with this, in humans, the enriched gut microbial diversity and beneficial species are responsible for the anti-inflammatory response and antiviral response (Uchiyama et al., 2019). Early exposure to a soil-rich natural environment has also been shown to increase gut microbiota diversity and beneficial microbes, and consequently enhance innate immunity and reduce respiratory tract infection of viral origin (Blum et al., 2019). Therefore, exposure to soilrich natural environments early in life may be useful to reduce the infection risk and avoid poor outcomes from COVID-19. Although it is mechanistically feasible, the role of early-life exposure to soil-rich natural environments in this setting has yet to be investigated in preclinical and clinical trials.

Early-life exposure to microbes may be vitally important, determining specific immune events that are durably imprinted into adulthood. With the progressive stabilization into an adult-like community structure throughout adulthood, the gut microbiota also plays an essential role in regulating immune responses to pathogenic viruses. For adults, specific gut microbiota-targeted approaches can be selected to increase the gut microbiota diversity and beneficial bacterial species. At present, the main examples of these gut 
microbiota-targeted approaches include FMT, probiotic supplementation, and the intake of products favoring the growth of beneficial bacteria and metabolite production, such as prebiotics and a Mediterranean-like diet (Figure 3).

Diet is the factor most strongly influencing the structure of the gut microbiota, exerting its influence throughout the lifespan. Published studies have consistently shown the ability of specific diets and dietary factors, mostly a Medi- terranean-like diet loaded with fermented food, dietary fibers, polyphenols, carotenoids, omega-3 polyunsaturated fatty acids, and unsaturated fatty acids, to preserve lung function and health (Scoditti et al., 2019). By contrast, a Western diet, rich in refined grains, desserts, sweets, sweetened beverages, saturated fat, cured meats (such as bacon, hot dogs, and processed meats), and red meats, is associated with impaired lung function and the risk of chronic ob-

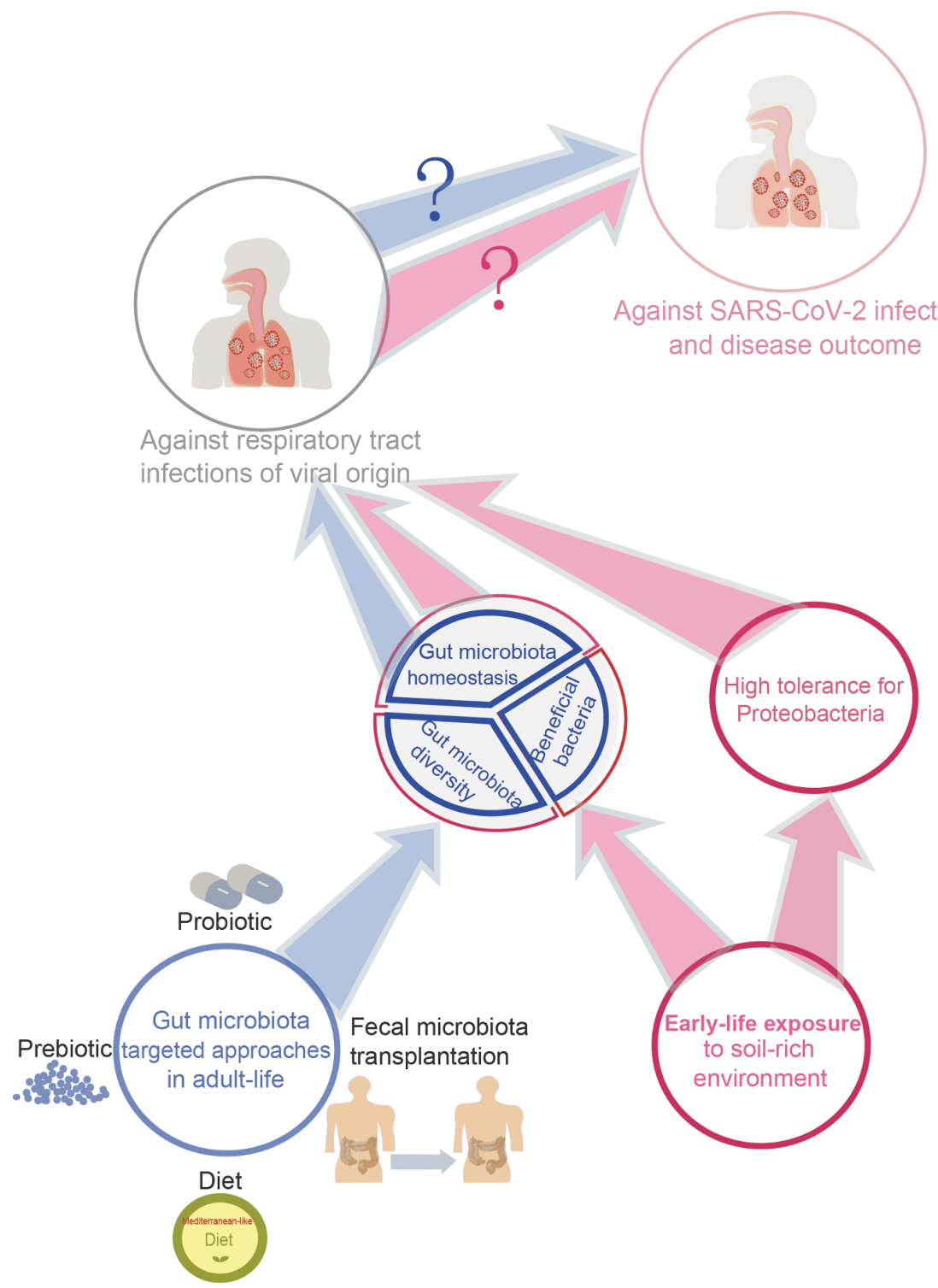

Figure 3 (Color online) Gut microbiota as a potential target against COVID-19. As exposure to specific microbes in early life plays an instrumental role in facilitating tolerance to their gut microbiota and following durable consequences for the immune response that extends into adult life, exposure to Proteobacteria in early life may promote a high tolerance for Proteobacteria (microbial signature of bats, centenarians and Hadza hunter-gathers) and following reduction in disease severity of viral infection. One of the environments dominated by Proteobacteria is the soil-rich natural environment. Moreover, early-life exposure to soil-rich natural environments maintains the gut microbiota homeostasis, increases the gut microbiota diversity and beneficial bacterial species, enhancing the immune defense to viral infection. In spite of the vital importance of early-life microbial exposures in determining specific immune events that are durably imprinted in adult life, the gut microbiota in adulthood also takes an essential part in regulating immune response to pathogenic virus. For adults, they can choose specific gut microbiota targeted approaches to maintain gut microbiota homeostasis, and increase the gut microbiota diversity and beneficial bacteria. These gut microbiota targeted approaches mainly include fecal microbiota transplantation (FMT), probiotic supplement and intake of products favoring the growth of beneficial bacteria and metabolite production, such as prebiotics and Mediterranean-like diets. Although the early-life exposure to soil-rich natural environments and these gut microbiota targeted approaches have a promising potential against respiratory tract infections of viral origin, the rationale for using them in COVID-19 is derived from indirect evidence, so their effect needs to be determined in future well-designed preclinical and clinical studies. 
structive pulmonary disease (Kaluza et al., 2019). Findings indicated that a Mediterranean diet improved lung health through eliciting favorable microbiota profiles and metabolite production, while a Western diet induced dysbiosis in gut microbiota composition, leading to elevated airway inflammation and reduced immune response against viral infection (Gentile and Weir, 2018). Among the Mediterraneanlike dietary factors, the dietary fibers can be fermented by gut microbiota to product SCFAs. SCFAs contribute to maintaining a healthy gut microbiota composition, and act on the gut-lung axis to promote lung health, including attenuating the inflammatory and allergic responses in the lungs, as well as enhancing the immune defense against respiratory viral infection (Dang and Marsland, 2019; Lewis et al., 2019). Besides the potential benefit of reducing the risk of SARSCoV-2 infection, SCFAs may also play a role in preventing pathogenic superinfection during SARS-CoV-2 infection. According to recent publications, alteration in the production of SCFAs by the gut microbiota increased susceptibility to secondary bacterial pneumonia in patients infected with the H7N9 influenza virus, while supplementation with SCFAs acetate reduced bacterial superinfection and lung pathology (Sencio et al., 2020). Bacterial superinfections can occur in the aftermath of viral infection and contribute significantly to the excess morbidity and mortality, as shown in influenza. In terms of COVID-19, the primary outcome measure is respiratory failure; the secondary outcome measure is the occurrence of bacterial superinfection among ventilated patients with SARS-CoV-2 pneumonia.

Prebiotics are non-digestible food ingredients that selectively stimulate the growth and activity of probiotics to improve host health. They mainly include oligosaccharides, unsaturated fatty acids, dietary fibers, and polyphenols (Gibson et al., 2017). Evidence suggests that, by improving the function of the microbiota-gut-lung axis, prebiotics can prevent respiratory diseases including respiratory tract infections, lung cancer, asthma, acute respiratory distress syndrome, chronic obstructive pulmonary disease, and acute lung injury (Huang et al., 2019; Langlois et al., 2019; Pizzini et al., 2018). Crucially, prebiotics, a high-fiber diet, and probiotics may have the potential to reduce the risk of severe symptoms in diabetic patients infected with SARS-CoV-2. Depending on the geographic region, $20 \%-50 \%$ of patients in the COVID-19 pandemic had diabetes. These patients have an increased risk of severe complications including respiratory distress syndrome and multi-organ failure, which may be attributable to high glucose levels (Bornstein et al., 2020). Coincidentally, high glucose levels may also explain why some patients with influenza infection have worse symptoms than others (Wang et al., 2020). Accumulated studies have demonstrated that prebiotics, dietary fiber, and probiotics can regulate glycometabolism by improving glucose control and insulin sensitivity (Anhê et al., 2015;
Cooper et al., 2017; Li et al., 2017).

The most commonly used probiotics are Lactobacillus and Bifidobacterium species. A number of animal and clinical studies have recently been reported that corroborate the effect of different probiotic strains to ameliorate or prevent viral infections, such as decreasing titers of Ebola and cytomegalovirus, and reduced duration and severity of respiratory tract infection caused by respiratory or influenza viruses (Dumas et al., 2018; Kanauchi et al., 2018; Kiousi et al., 2019). For instance, one study revealed that Lactobacillus gasseri can protect against respiratory syncytial virus infection in mice, as the RSV titer and expression of proinflammatory cytokines in the lungs were significantly decreased, while IFN and ISGs were upregulated after the treatment (Eguchi et al., 2019). With regard to clinical trials, probiotic consumption has been shown to be effective at reducing the severity and duration of respiratory tract infections and increasing the immune defense against influenza in children (Laursen and Hojsak, 2018; Wang et al., 2016) adults, and the elderly (Lehtoranta et al., 2014). However, it should be noted that the beneficial effects of probiotics on the outcome of respiratory tract infections may be dependent on the use of different probiotic strains, bacterial dose, and the matrices used.

Fecal microbiota transplantation (FMT) is the process by which fecal microbiota is transplanted from a healthy donor to the recipient's gut in order to recover gut microbial homeostasis. It has been effectively used in the treatment of various diseases including recurrent Clostridium difficile infection, IBD, IBS, and obesity (Wortelboer et al., 2019). Remodeling the gut microbiota through FMT not only recovers gastrointestinal function, but is also effective at treating respiratory tract diseases. The latest research has indicated that FMT can be beneficial at relieving respiratory tract inflammation, ameliorating viral and bacterial infection (Jagessar et al., 2019; Laffin et al., 2017), and improving endotoxic acute lung injury ( $\mathrm{Li}$ et al., 2020a).

The above data suggest that probiotic supplementation and FMT have potential for use against respiratory tract infections of viral origin. However, no reported studies have yet evaluated the use of probiotics and FMT as add-on therapy for COVID-19. Similarly, at present, there is only indirect evidence supporting the use of dietary fiber and prebiotics as daily supplements in the personalized diet in COVID-19 patients. Therefore, the application of these gut microbiotatargeted approaches to reduce infection and improve clinical outcomes of COVID-19 need further support from evidence from preclinical and clinical trials (Figure 3).

\section{Conclusion}

Mammals have evolved in a microbial world, so gut mi- 
crobial symbionts have played a vital role in antiviral defense to promote host health. The antiviral role of gut microbiota has been well studied in the laboratory. To date, the majority of such studies have focused on laboratory mouse models, being confined to highly sterile environments, which is not representative of the complexity of gut microbiota present in wild hosts and results in altered immune development (Rosshart et al., 2019). Thus, these laboratory studies have left broad patterns of gut microbial function and natural immune response to viruses unclear. In gut microbiota research, there is an urgent need for a wildlife model system. Bats are unique among mammals as the only ones capable of hosting and co-existing with various viruses, including some of the world's deadliest viruses and those that infect humans. We propose that bats represent a model system ideal for comparative microbiome research for human infectious disease of viral origin, which can help us answer key questions about viral tolerance and disease outcome. In addition, bats include an abundance of species and enjoy a cosmopolitan global distribution, with nearly 1,400 described species, constituting approximately $20 \%$ of all living mammals, and being found on every continent save Antarctica. As a highly diverse and ecologically important mammal, bats are a good system within which to attempt to answer new questions about the gut microbiota-driven virus-host coevolutionary trajectory.

In its adaptation to a flight-based lifestyle, the unique gut microbial composition profoundly contributes to bats' status as a viral reservoir. In future studies, there is a need to clarify the molecular pathways by which this special gut microbiota influences the tolerance of bats to viruses and the speciesbased or community-based effects. It is clear that such studies will shed light on how the gut microbiota impacts on infectious disease susceptibility and outcome in bats and humans. For instance, since the gut microbiota of bats is more closely linked to their living habitats, more attention should be paid to the possibility that human-caused disturbances to bats' habitat may perturb their gut microbiota, such as by destroying their immune defense against white nose syndrome, which has ravaged bat colonies all over the world. As for humans, advances in our understanding of batmicrobe mutualism may highlight how to avoid severe disease outcomes in SARS-CoV-2 infection.

Given the importance of gut microbiota in immune defense against viral infection, there is a need for caution to avoid unnecessary antibiotic treatments. With significant adverse effects on gut microbiota homeostasis and diversity, increased antibiotic use may have the unintended and devastating consequence of increasing the risk for SARS-CoV-2 infection and severe disease outcome. However, in China, $58 \%-71 \%$ of COVID- 19 patients were given antibiotics, and diarrhea occurred in $2 \%-36 \%$ of patients (Guan et al., 2020; Wong et al., 2020). Hopefully, the application of these findings in a clinical context is approaching. When antibiotics are used in COVID-19 patients, the re-establishment of gut microbiota homeostasis by probiotic supplementation to reduce infection and fatal outcome has been proposed globally. China's National Health Commission and National Administration of Traditional Chinese Medicine have suggested the use of probiotics in patients with severe COVID19. In the United Kingdom, an ongoing Phase II randomized, double-blind, placebo-controlled trial is evaluating the efficacy and safety of oral live biotherapeutic MRx-4DP0004 in addition to standard supportive care for hospitalized COVID-19 patients. In the USA, a multicenter, Phase II randomized, double-blind, placebo-controlled trial is being conducted to evaluate the ability of a commercial product of Lactobacillus rhamnosus GG to decrease infection and improve outcome in COVID-19 patients (Akour, 2020).

Besides highlighting the gut microbiota per se in COVID19 , future studies may also explore the effect of microbiotaderived metabolites, such as fatty acids, neural signals, and polyamines. Among these, SCFAs have emerged as key signaling molecules within the gut and in the periphery to limit inflammation and enhance protective responses to viral infection. Second, further studies should clarify whether the lung microbiota is involved in COVID-19. Cross-talk between the gut and lung microbiota has been proposed, as it showed that changes at one of these two sites could impact the other. Finally, it would be interesting to dissect the role of other gut microorganisms such as fungi and other viruses in COVID-19. In fact, fungi and viruses form an important niche ecosystem in the gut, which respectively constitute the gut mycobiome and the gut virome. A recent report showed that COVID-19 patients had significant alterations in their fecal mycobiome compared with controls, characterized by enrichment of Candida albicans and a highly heterogeneous mycobiome configuration, at the time of hospitalization, which persisted in a subset of patients for up to 12 days after nasopharyngeal clearance of SARS-CoV-2. However, the contributions of an altered gut mycobiome to SARS-CoV-2 infection and disease progression need to be determined in future work (Zuo et al., 2020b).

Compliance and ethics The author(s) declare that they have no conflict of interest.

\section{References}

Ahn, M., Anderson, D.E., Zhang, Q., Tan, C.W., Lim, B.L., Luko, K., Wen, M., Chia, W.N., Mani, S., Wang, L.C., et al. (2019). Dampened NLRP3mediated inflammation in bats and implications for a special viral reservoir host. Nat Microbiol 4, 789-799.

Akour, A. (2020). Probiotics and COVID-19: is there any link? Lett Appl Microbiol 71, 229-234.

AlGhatrif, M., Cingolani, O., and Lakatta, E.G. (2020). The dilemma of coronavirus disease 2019, aging, and cardiovascular disease. JAMA Cardiol 5, 747-748.

Andersen, K.G., Rambaut, A., Lipkin, W.I., Holmes, E.C., and Garry, R.F. 
(2020). The proximal origin of SARS-CoV-2. Nat Med 26, 450-452.

Anhê, F.F., Roy, D., Pilon, G., Dudonné, S., Matamoros, S., Varin, T.V., Garofalo, C., Moine, Q., Desjardins, Y., Levy, E., et al. (2015). A polyphenol-rich cranberry extract protects from diet-induced obesity, insulin resistance and intestinal inflammation in association with increased Akkermansia spp. population in the gut microbiota of mice. Gut 64, 872-883.

Asadi-Pooya, A.A., and Simani, L. (2020). Central nervous system manifestations of COVID-19: a systematic review. J Neurol Sci 413, 116832

Banerjee, A., Baker, M.L., Kulcsar, K., Misra, V., Plowright, R., and Mossman, K. (2020). Novel insights into immune systems of bats. Front Immunol 11 .

Banerjee, A., Rapin, N., Bollinger, T., and Misra, V. (2017). Lack of inflammatory gene expression in bats: a unique role for a transcription repressor. Sci Rep 7, 2232.

Battaglini, D., Pimentel-Coelho, P.M., Robba, C., Dos Santos, C.C., Cruz, F.F., Pelosi, P., and Rocco, P.R.M. (2020). Gut microbiota in acute ischemic stroke: from pathophysiology to therapeutic implications. Front Neurol 11

Beyrouti, R., Adams, M.E., Benjamin, L., Cohen, H., Farmer, S.F., Goh, Y. Y., Humphries, F., Jäger, H.R., Losseff, N.A., Perry, R.J., et al. (2020). Characteristics of ischaemic stroke associated with COVID-19. J Neurol Neurosurg Psychiatry 91, 889-891.

Biagi, E., Rampelli, S., Turroni, S., Quercia, S., Candela, M., and Brigidi, P. (2017). The gut microbiota of centenarians: signatures of longevity in the gut microbiota profile. Mech Ageing Dev 165, 180-184.

Blum, W.E.H., Zechmeister-Boltenstern, S., and Keiblinger, K.M. (2019). Does soil contribute to the human gut microbiome? Microorganisms 7, 287.

Bornstein, S.R., Rubino, F., Khunti, K., Mingrone, G., Hopkins, D., Birkenfeld, A.L., Boehm, B., Amiel, S., Holt, R.I., Skyler, J.S., et al. (2020). Practical recommendations for the management of diabetes in patients with COVID-19. Lancet Diabetes Endocrinol 8, 546-550.

Bradley, K.C., Finsterbusch, K., Schnepf, D., Crotta, S., Llorian, M., Davidson, S., Fuchs, S.Y., Staeheli, P., and Wack, A. (2019) Microbiota-driven tonic interferon signals in lung stromal cells protect from influenza virus infection. Cell Rep 28, 245-256.e4.

Callaway, E. (2020). Labs rush to study coronavirus in transgenic animals - some are in short supply. Nature 579, 183.

Caviedes-Vidal, E., McWhorter, T.J., Lavin, S.R., Chediack, J.G., Tracy, C. R., and Karasov, W.H. (2007). The digestive adaptation of flying vertebrates: high intestinal paracellular absorption compensates for smaller guts. Proc Natl Acad Sci USA 104, 19132-19137.

Chen, L., and Zhong, L. (2020). Lung adenocarcinoma patients own higher risk of SARS-CoV-2 infection. Preprints, 2020020386.

Chen, X., Laurent, S., Onur, O.A., Kleineberg, N.N., Fink, G.R., Schweitzer, F., and Warnke, C. (2020). A systematic review of neurological symptoms and complications of COVID-19. J Neurol, doi: 10.1007/s00415-020-10067-3.

Cooper, D.N., Kable, M.E., Marco, M.L., De Leon, A., Rust, B., Baker, J. E., Horn, W., Burnett, D., and Keim, N.L. (2017). The effects of moderate whole grain consumption on fasting glucose and lipids, gastrointestinal symptoms, and microbiota. Nutrients 9, 173.

Cryan, J.F., O'Riordan, K.J., Cowan, C.S.M., Sandhu, K.V., Bastiaanssen, T.F.S., Boehme, M., Codagnone, M.G., Cussotto, S., Fulling, C., Golubeva, A.V., et al. (2019). The microbiota-gut-brain axis. Physiol Rev 99, 1877-2013.

Dang, A.T., and Marsland, B.J. (2019). Microbes, metabolites, and the gutlung axis. Mucosal Immunol 12, 843-850.

De Filippo, C., Cavalieri, D., Di Paola, M., Ramazzotti, M., Baptiste Poullet, J., Massart, S., Collini, S., Pieraccini, G., and Lionetti, P. (2010). Impact of diet in shaping gut microbiota revealed by a comparative study in children from Europe and rural Africa. Proc Natl Acad Sci USA 107, 14691-14696.

del Campo, R., Garriga, M., Pérez-Aragón, A., Guallarte, P., Lamas, A., Máiz, L., Bayón, C., Roy, G., Cantón, R., Zamora, J., et al. (2014).
Improvement of digestive health and reduction in proteobacterial populations in the gut microbiota of cystic fibrosis patients using a Lactobacillus reuteri probiotic preparation: a double blind prospective study. J Cystic Fibrosis 13, 716-722.

Du, R.H., Liang, L.R., Yang, C.Q., Wang, W., Cao, T.Z., Li, M., Guo, G.Y., Du, J., Zheng, C.L., Zhu, Q., et al. (2020). Predictors of mortality for patients with COVID-19 pneumonia caused by SARS-CoV-2: a prospective cohort study. Eur Respir J 55, 2000524.

Dumas, A., Bernard, L., Poquet, Y., Lugo-Villarino, G., and Neyrolles, O. (2018). The role of the lung microbiota and the gut-lung axis in respiratory infectious diseases. Cell Microbiol 20, e12966.

Eguchi, K., Fujitani, N., Nakagawa, H., and Miyazaki, T. (2019). Prevention of respiratory syncytial virus infection with probiotic lactic acid bacterium Lactobacillus gasseri SBT2055. Sci Rep 9, 4812.

Eom, T., Kim, Y.S., Choi, C.H., Sadowsky, M.J., and Unno, T. (2018). Current understanding of microbiota- and dietary-therapies for treating inflammatory bowel disease. J Microbiol 56, 189-198.

Fang, L., Karakiulakis, G., and Roth, M. (2020). Are patients with hypertension and diabetes mellitus at increased risk for COVID-19 infection? Lancet Respir Med 8, e21.

Finer, N., Garnett, S.P., and Bruun, J.M. (2020). COVID-19 and obesity. Clin Obes 10, e12365.

Follmer, C. (2020). Gut microbiome imbalance and neuroinflammation: impact of COVID-19 on Parkinson's disease. Mov Disord 35, 1495 1496.

Franceschi, C., Capri, M., Monti, D., Giunta, S., Olivieri, F., Sevini, F., Panourgia, M.P., Invidia, L., Celani, L., Scurti, M., et al. (2007). Inflammaging and anti-inflammaging: a systemic perspective on aging and longevity emerged from studies in humans. Mech Ageing Dev 128, 92-105.

Francino, M.P. (2016). Antibiotics and the human gut microbiome: dysbioses and accumulation of resistances. Front Microbiol 6, 1543.

Fransen, F., van Beek, A.A., Borghuis, T., Aidy, S.E., Hugenholtz, F., van der Gaast-de Jongh, C., Savelkoul, H.F.J., De Jonge, M.I., Boekschoten, M.V., Smidt, H., et al. (2017). Aged gut microbiota contributes to systemical inflammaging after transfer to germ-free mice. Front Immunol 8, 1385.

Franzosa, E.A., Sirota-Madi, A., Avila-Pacheco, J., Fornelos, N., Haiser, H. J., Reinker, S., Vatanen, T., Hall, A.B., Mallick, H., McIver, L.J., et al. (2019). Gut microbiome structure and metabolic activity in inflammatory bowel disease. Nat Microbiol 4, 293-305.

Gensollen, T., Iyer, S.S., Kasper, D.L., and Blumberg, R.S. (2016). How colonization by microbiota in early life shapes the immune system. Science 352, 539-544.

Gentile, C.L., and Weir, T.L. (2018). The gut microbiota at the intersection of diet and human health. Science 362, 776-780.

Gibson, G.R., Hutkins, R., Sanders, M.E., Prescott, S.L., Reimer, R.A., Salminen, S.J., Scott, K., Stanton, C., Swanson, K.S., Cani, P.D., et al. (2017). Expert consensus document: the international scientific association for probiotics and prebiotics (ISAPP) consensus statement on the definition and scope of prebiotics. Nat Rev Gastroenterol Hepatol 14, 491-502.

Guan, W.J., Ni, Z.Y., Hu, Y., Liang, W.H., Ou, C.Q., He, J.X., Liu, L., Shan, H., Lei, C.L., Hui, D.S.C., et al. (2020). Clinical characteristics of coronavirus disease 2019 in China. N Engl J Med 382, 1708-1720.

Hölzer, M., Schoen, A., Wulle, J., Müller, M.A., Drosten, C., Marz, M., and Weber, F. (2019). Virus- and interferon alpha-induced transcriptomes of cells from the microbat Myotis daubentonii. iScience 19, 647-661.

Hashimoto, T., Perlot, T., Rehman, A., Trichereau, J., Ishiguro, H., Paolino, M., Sigl, V., Hanada, T., Hanada, R., Lipinski, S., et al. (2012). ACE2 links amino acid malnutrition to microbial ecology and intestinal inflammation. Nature 487, 477-481.

Henry, B.M., and Lippi, G. (2020). Chronic kidney disease is associated with severe coronavirus disease 2019 (COVID-19) infection. Int Urol Nephrol 52, 1193-1194.

Hills, R.D., Pontefract, B.A., Mishcon, H.R., Black, C.A., Sutton, S.C., and Theberge, C.R. (2019). Gut microbiome: profound implications for diet 
and disease. Nutrients 11,1613

Honda, K., and Littman, D.R. (2016). The microbiota in adaptive immune homeostasis and disease. Nature 535, 75-84.

Huang, L., Guo, J., Li, W., Jiang, M., Wang, F., Kang, J., Liu, T., and Gu, X. (2019). Probiotics, prebiotics, and synbiotics for the treatment of asthma. Medicine 98, e17840.

Huang, Y., Mao, K., Chen, X., Sun, M., Kawabe, T., Li, W., Usher, N., Zhu, J., Urban Jr., J.F., Paul, W.E., et al. (2018). S1P-dependent interorgan trafficking of group 2 innate lymphoid cells supports host defense. Science 359, 114-119.

Ianiro, G., Tilg, H., and Gasbarrini, A. (2016). Antibiotics as deep modulators of gut microbiota: between good and evil. Gut 65, 19061915.

Ingala, M.R., Becker, D.J., Bak Holm, J., Kristiansen, K., and Simmons, N. B. (2019). Habitat fragmentation is associated with dietary shifts and microbiota variability in common vampire bats. Ecol Evol 9, 65086523.

Jagessar, S.A.R., Long, C., Cui, B., and Zhang, F. (2019). Improvement of Good's syndrome by fecal microbiota transplantation: the first case report. J Int Med Res 47, 3408-3415.

Kaluza, J., Harris, H., Linden, A., and Wolk, A. (2019). Long-term unprocessed and processed red meat consumption and risk of chronic obstructive pulmonary disease: a prospective cohort study of women. Eur J Nutr 58, 665-672.

Kanauchi, O., Andoh, A., AbuBakar, S., and Yamamoto, N. (2018). Probiotics and paraprobiotics in viral infection: clinical application and effects on the innate and acquired immune systems. Curr Pharm Design 24, 710-717.

Kempuraj, D., Selvakumar, G.P., Ahmed, M.E., Raikwar, S.P., Thangavel, R., Khan, A., Zaheer, S.A., Iyer, S.S., Burton, C., James, D., et al. (2020). COVID-19, mast cells, cytokine storm, psychological stress, and neuroinflammation. Neuroscientist 26, 402-414.

Kim, S., Rigatto, K., Gazzana, M.B., Knorst, M.M., Richards, E.M., Pepine, C.J., and Raizada, M.K. (2020). Altered gut microbiome profile in patients with pulmonary arterial hypertension. Hypertension 75, 1063-1071.

Kiousi, D.E., Karapetsas, A., Karolidou, K., Panayiotidis, M.I., Pappa, A., and Galanis, A. (2019). Probiotics in extraintestinal diseases: current trends and new directions. Nutrients 11, 788.

Laffin, M., Millan, B., and Madsen, K.L. (2017). Fecal microbial transplantation as a therapeutic option in patients colonized with antibiotic resistant organisms. Gut Microbes 8, 221-224.

Langlois, P.L., D'Aragon, F., Hardy, G., and Manzanares, W. (2019). Omega-3 polyunsaturated fatty acids in critically ill patients with acute respiratory distress syndrome: a systematic review and meta-analysis. Nutrition 61, 84-92.

Laursen, R.P., and Hojsak, I. (2018). Probiotics for respiratory tract infections in children attending day care centers - a systematic review. Eur J Pediatr 177, 979-994.

Lehtoranta, L., Pitkäranta, A., and Korpela, R. (2014). Probiotics in respiratory virus infections. Eur J Clin Microbiol Infect Dis 33, 12891302.

Letko, M., Marzi, A., and Munster, V. (2020). Functional assessment of cell entry and receptor usage for SARS-CoV-2 and other lineage B betacoronaviruses. Nat Microbiol 5, 562-569.

Lewis, G., Wang, B., Shafiei Jahani, P., Hurrell, B.P., Banie, H., Aleman Muench, G.R., Maazi, H., Helou, D.G., Howard, E., Galle-Treger, L., et al. (2019). Dietary fiber-induced microbial short chain fatty acids suppress ILC2-dependent airway inflammation. Front Immunol 10, 2051.

Li, B., Yin, G.F., Wang, Y.L., Tan, Y.M., Huang, C.L., and Fan, X.M. (2020a). Impact of fecal microbiota transplantation on TGF- $\beta 1 /$ Smads/ ERK signaling pathway of endotoxic acute lung injury in rats. 3 Biotech $10,52$.

Li, G., He, X., Zhang, L., Ran, Q., Wang, J., Xiong, A., Wu, D., Chen, F., Sun, J., and Chang, C. (2020b). Assessing ACE2 expression patterns in lung tissues in the pathogenesis of COVID-19. J Autoimmun 112,
102463.

Li, J., Li, L., Jiang, H., Yuan, L., Zhang, L., Ma, J.E., Zhang, X., Cheng, M., and Chen, J. (2018). Fecal bacteriome and mycobiome in bats with diverse diets in South China. Curr Microbiol 75, 1352-1361.

Li, M., Dai, B., Tang, Y., Lei, L., Li, N., Liu, C., Ge, T., Zhang, L., Xu, Y., $\mathrm{Hu}$, Y., et al. (2019). Altered bacterial-fungal interkingdom networks in the guts of ankylosing spondylitis patients. mSystems 4 .

Li, T., Tang, X., Wu, C., Yao, X., Wang, Y., Lu, X., and Lu, J. (2020c). The use of SARS-CoV-2-related coronaviruses from bats and pangolins to polarize mutations in SARS-Cov-2. Sci China Life Sci 63, 1608-1611.

Li, X., Wang, E., Yin, B., Fang, D., Chen, P., Wang, G., Zhao, J., Zhang, H., and Chen, W. (2017). Effects of Lactobacillus casei CCFM419 on insulin resistance and gut microbiota in type 2 diabetic mice. Benef Microbes 8, 421-432.

Liang, W., Feng, Z., Rao, S., Xiao, C., Xue, X., Lin, Z., Zhang, Q., and Qi, W. (2020). Diarrhoea may be underestimated: a missing link in 2019 novel coronavirus. Gut 69, 1141-1143.

Lin, C.H., Chen, C.C., Chiang, H.L., Liou, J.M., Chang, C.M., Lu, T.P., Chuang, E.Y., Tai, Y.C., Cheng, C., Lin, H.Y., et al. (2019). Altered gut microbiota and inflammatory cytokine responses in patients with Parkinson's disease. J Neuroinflamm 16, 129.

Lutz, H.L., Jackson, E.W., Webala, P.W., Babyesiza, W.S., Kerbis Peterhans, J.C., Demos, T.C., Patterson, B.D., and Gilbert, J.A. (2019). Ecology and host identity outweigh evolutionary history in shaping the bat microbiome. mSystems 4 .

Marsland, B.J., Trompette, A., and Gollwitzer, E.S. (2015). The gut-lung axis in respiratory disease. Ann Am Thorac Soc 12, S150-S156.

Matenchuk, B.A., Mandhane, P.J., and Kozyrskyj, A.L. (2020). Sleep, circadian rhythm, and gut microbiota. Sleep Med Rev 53, 101340.

McAleer, J.P., and Kolls, J.K. (2018). Contributions of the intestinal microbiome in lung immunity. Eur J Immunol 48, 39-49.

Mu, J., Xu, J., Zhang, L., Shu, T., Wu, D., Huang, M., Ren, Y., Li, X., Geng, Q., Xu, Y., et al. (2020). SARS-CoV-2-encoded nucleocapsid protein acts as a viral suppressor of RNA interference in cells. Sci China Life Sci 63, 1-4.

Muccioli, L., Pensato, U., Cani, I., Guarino, M., Cortelli, P., and Bisulli, F. (2020). COVID-19-associated encephalopathy and cytokine-mediated neuroinflammation. Ann Neurol 88, 860-861.

O’Sullivan, O., Cronin, O., Clarke, S.F., Murphy, E.F., Molloy, M.G., Shanahan, F., and Cotter, P.D. (2015). Exercise and the microbiota. Gut Microbes 6, 131-136.

O’Toole, P.W., and Jeffery, I.B. (2015). Gut microbiota and aging. Science 350, 1214-1215.

Odamaki, T., Kato, K., Sugahara, H., Hashikura, N., Takahashi, S., Xiao, J. Z., Abe, F., and Osawa, R. (2016). Age-related changes in gut microbiota composition from newborn to centenarian: a cross-sectional study. BMC Microbiol 16, 90.

Pizzini, A., Lunger, L., Sonnweber, T., Weiss, G., and Tancevski, I. (2018). The role of omega- 3 fatty acids in the setting of coronary artery disease and COPD: a review. Nutrients 10, 1864.

Prins, G.H., and Olinga, P. (2020). Potential implications of COVID-19 in non-alcoholic fatty liver disease. Liver Int 40, 2568.

Qian, Q., Fan, L., Liu, W., Li, J., Yue, J., Wang, M., Ke, X., Yin, Y., Chen, Q., and Jiang, C. (2020). Direct evidence of active SARS-CoV-2 replication in the intestine. Clin Infect Dis, doi: 10.1093/cid/ciaa1925.

Rampelli, S., Schnorr, S.L., Consolandi, C., Turroni, S., Severgnini, M., Peano, C., Brigidi, P., Crittenden, A.N., Henry, A.G., and Candela, M. (2015). Metagenome sequencing of the Hadza hunter-gatherer gut microbiota. Curr Biol 25, 1682-1693.

Rinninella, E., Raoul, P., Cintoni, M., Franceschi, F., Miggiano, G.A.D., Gasbarrini, A., and Mele, M.C. (2019). What is the healthy gut microbiota composition? A changing ecosystem across age, environment, diet, and diseases. Microorganisms 7, 14.

Rosser, E.C., Oleinika, K., Tonon, S., Doyle, R., Bosma, A., Carter, N.A., Harris, K.A., Jones, S.A., Klein, N., and Mauri, C. (2014). Regulatory B cells are induced by gut microbiota-driven interleukin-1 $\beta$ and interleukin-6 production. Nat Med 20, 1334-1339. 
Rosshart, S.P., Herz, J., Vassallo, B.G., Hunter, A., Wall, M.K., Badger, J. H., McCulloch, J.A., Anastasakis, D.G., Sarshad, A.A., Leonardi, I., et al. (2019). Laboratory mice born to wild mice have natural microbiota and model human immune responses. Science 365, eaaw4361.

Rothschild, D., Weissbrod, O., Barkan, E., Kurilshikov, A., Korem, T., Zeevi, D., Costea, P.I., Godneva, A., Kalka, I.N., Bar, N., et al. (2018). Environment dominates over host genetics in shaping human gut microbiota. Nature 555, 210-215.

Sampson, T.R., Debelius, J.W., Thron, T., Janssen, S., Shastri, G.G., Ilhan, Z.E., Challis, C., Schretter, C.E., Rocha, S., Gradinaru, V., et al. (2016). Gut microbiota regulate motor deficits and neuroinflammation in a model of Parkinson's disease. Cell 167, 1469-1480.e12.

Santoro, A., Ostan, R., Candela, M., Biagi, E., Brigidi, P., Capri, M., and Franceschi, C. (2018). Gut microbiota changes in the extreme decades of human life: a focus on centenarians. Cell Mol Life Sci 75, 129-148.

Schnorr, S.L., Candela, M., Rampelli, S., Centanni, M., Consolandi, C., Basaglia, G., Turroni, S., Biagi, E., Peano, C., Severgnini, M., et al. (2014). Gut microbiome of the Hadza hunter-gatherers. Nat Commun 5, 3654 .

Scoditti, E., Massaro, M., Garbarino, S., and Toraldo, D.M. (2019). Role of diet in chronic obstructive pulmonary disease prevention and treatment. Nutrients 11, 1357.

Sencio, V., Barthelemy, A., Tavares, L.P., Machado, M.G., Soulard, D., Cuinat, C., Queiroz-Junior, C.M., Noordine, M.L., Salomé-Desnoulez, S., Deryuter, L., et al. (2020). Gut dysbiosis during influenza contributes to pulmonary pneumococcal superinfection through altered short-chain fatty acid production. Cell Rep 30, 2934-2947.e6.

Seo, S.U., Kamada, N., Muñoz-Planillo, R., Kim, Y.G., Kim, D., Koizumi, Y., Hasegawa, M., Himpsl, S.D., Browne, H.P., Lawley, T.D., et al. (2015). Distinct commensals induce interleukin-1 $\beta$ via NLRP3 inflammasome in inflammatory monocytes to promote intestinal inflammation in response to injury. Immunity 42, 744-755.

Shao, Y., Forster, S.C., Tsaliki, E., Vervier, K., Strang, A., Simpson, N., Kumar, N., Stares, M.D., Rodger, A., Brocklehurst, P., et al. (2019). Stunted microbiota and opportunistic pathogen colonization in caesarean-section birth. Nature 574, 117-121.

Shin, N.R., Whon, T.W., and Bae, J.W. (2015). Proteobacteria: microbial signature of dysbiosis in gut microbiota. Trends Biotech 33, 496-503.

Gu, S., Chen, Y., Wu, Z., Chen, Y., Gao, H., Lv, L., Guo, F., Zhang, X., Luo, R., Huang, C., et al. (2020). Alterations of the gut microbiota in patients with coronavirus disease 2019 or H1N1 influenza. Clin Infect Dis 71, 2669-2678.

Singh, V., Yeoh, B.S., Walker, R.E., Xiao, X., Saha, P., Golonka, R.M., Cai, J., Bretin, A.C.A., Cheng, X., Liu, Q., et al. (2019). Microbiota fermentation-NLRP3 axis shapes the impact of dietary fibres on intestinal inflammation. Gut 68, 1801-1812.

Song, S.J., Sanders, J.G., Delsuc, F., Metcalf, J., Amato, K., Taylor, M.W., Mazel, F., Lutz, H.L., Winker, K., Graves, G.R., et al. (2020a). Comparative analyses of vertebrate gut microbiomes reveal convergence between birds and bats. mBio 11, e02901-02919.

Song, Y., Liu, P., Shi, X.L., Chu, Y.L., Zhang, J., Xia, J., Gao, X.Z., Qu, T., and Wang, M.Y. (2020b). SARS-CoV-2 induced diarrhoea as onset symptom in patient with COVID-19. Gut 69, 1143-1144.

Stanifer, M.L., Kee, C., Cortese, M., Zumaran, C.M., Triana, S., Mukenhirn, M., Kraeusslich, H.G., Alexandrov, T., Bartenschlager, R., and Boulant, S. (2020). Critical role of type III interferon in controlling SARS-CoV-2 infection in human intestinal epithelial cells. Cell Rep 32, 107863.

Steed, A.L., Christophi, G.P., Kaiko, G.E., Sun, L., Goodwin, V.M., Jain, U., Esaulova, E., Artyomov, M.N., Morales, D.J., Holtzman, M.J., et al. (2017). The microbial metabolite desaminotyrosine protects from influenza through type I interferon. Science 357, 498-502.

Subudhi, S., Rapin, N., and Misra, V. (2019). Immune system modulation and viral persistence in bats: understanding viral spillover. Viruses 11 , 192.

Sultana, S., and Ananthapur, V. (2020). COVID-19 and its impact on neurological manifestations and mental health: the present scenario.
Neurol Sci, 1-6, doi: 10.1007/s10072-10020-04695-w.

Sun, M.F., Zhu, Y.L., Zhou, Z.L., Jia, X.B., Xu, Y.D., Yang, Q., Cui, C., and Shen, Y.Q. (2018). Neuroprotective effects of fecal microbiota transplantation on MPTP-induced Parkinson's disease mice: Gut microbiota, glial reaction and TLR4/TNF- $\alpha$ signaling pathway. Brain Behav Immun 70, 48-60.

Tasnim, N., Abulizi, N., Pither, J., Hart, M.M., and Gibson, D.L. (2017). Linking the gut microbial ecosystem with the environment: does gut health depend on where we live? Front Microbiol 8.

Thaiss, C.A., Zmora, N., Levy, M., and Elinav, E. (2016). The microbiome and innate immunity. Nature 535, 65-74.

Thevaranjan, N., Puchta, A., Schulz, C., Naidoo, A., Szamosi, J.C., Verschoor, C.P., Loukov, D., Schenck, L.P., Jury, J., Foley, K.P., et al. (2017). Age-associated microbial dysbiosis promotes intestinal permeability, systemic inflammation, and macrophage dysfunction. Cell Host Microbe 21, 455-466.e4.

Tomova, A., Bukovsky, I., Rembert, E., Yonas, W., Alwarith, J., Barnard, N.D., and Kahleova, H. (2019). The effects of vegetarian and vegan diets on gut microbiota. Front Nutr 6, 47.

Torres, J., and Peter, I. (2019). Neonatal exposures and risk of inflammatory bowel disease: when does the clock start ticking? Gastroenterology 157, 577-578.

Trompette, A., Gollwitzer, E.S., Yadava, K., Sichelstiel, A.K., Sprenger, N., Ngom-Bru, C., Blanchard, C., Junt, T., Nicod, L.P., Harris, N.L., et al. (2014). Gut microbiota metabolism of dietary fiber influences allergic airway disease and hematopoiesis. Nat Med 20, 159-166.

Uchiyama, K., Naito, Y., and Takagi, T. (2019). Intestinal microbiome as a novel therapeutic target for local and systemic inflammation. Pharmacol Therapeutics 199, 164-172.

Wang, L.F., and Anderson, D.E. (2019). Viruses in bats and potential spillover to animals and humans. Curr Opin Virol 34, 79-89.

Wang, Q., Fang, P., He, R., Li, M., Yu, H., Zhou, L., Yi, Y., Wang, F., Rong, Y., Zhang, Y., et al. (2020). O-GlcNAc transferase promotes influenza A virus-induced cytokine storm by targeting interferon regulatory factor5. Sci Adv 6, eaaz7086.

Wang, Y., Li, X., Ge, T., Xiao, Y., Liao, Y., Cui, Y., Zhang, Y., Ho, W., Yu, G., and Zhang, T. (2016). Probiotics for prevention and treatment of respiratory tract infections in children. Medicine 95, e4509.

Wasimuddin, , Brändel, S.D., Tschapka, M., Page, R., Rasche, A., Corman, V.M., Drosten, C., and Sommer, S. (2018). Astrovirus infections induce age-dependent dysbiosis in gut microbiomes of bats. ISME J 12, 28832893.

Whittaker, A., Anson, M., and Harky, A. (2020). Neurological manifestations of COVID-19: a systematic review and current update. Acta Neurol Scand 142, 14-22.

Wong, S.H., Lui, R.N., and Sung, J.J. (2020). COVID-19 and the digestive system. J Gastroenterol Hepatol 35, 744-748.

Wortelboer, K., Nieuwdorp, M., and Herrema, H. (2019). Fecal microbiota transplantation beyond Clostridioides difficile infections. EBioMedicine 44, 716-729.

Xiao, F., Tang, M., Zheng, X., Liu, Y., Li, X., and Shan, H. (2020). Evidence for gastrointestinal infection of SARS-CoV-2. Gastroenterology 158, 1831-1833.e3.

Xie, J., Li, Y., Shen, X., Goh, G., Zhu, Y., Cui, J., Wang, L.F., Shi, Z.L., and Zhou, P. (2018). Dampened STING-dependent interferon activation in bats. Cell Host Microbe 23, 297-301.e4.

Xu, K., Cai, H., Shen, Y., Ni, Q., and Li, L. (2020). Management of corona virus disease-19 (COVID-19): the Zhejiang experience (in Chinese). J Zhejiang Univ Med Sci 49.

Yang, D., Zhao, D., Ali Shah, S.Z., Wu, W., Lai, M., Zhang, X., Li, J., Guan, Z., Zhao, H., Li, W., et al. (2019). The Role of the Gut Microbiota in the Pathogenesis of Parkinson's Disease. Front Neurol 10.

Yeo, C., Kaushal, S., and Yeo, D. (2020). Enteric involvement of coronaviruses: is faecal-oral transmission of SARS-CoV-2 possible? Lancet Gastroenterology Hepatology 5, 335-337.

Yuan, Y., Wang, N., and Ou, X. (2020). Caution should be exercised for the detection of SARS-CoV-2, especially in the elderly. J Med Virol 92, 
$1641-1648$

Zepeda Mendoza, M.L., Xiong, Z., Escalera-Zamudio, M., Runge, A.K., Thézé, J., Streicker, D., Frank, H.K., Loza-Rubio, E., Liu, S., Ryder, O. A., et al. (2018). Hologenomic adaptations underlying the evolution of sanguivory in the common vampire bat. Nat Ecol Evol 2, 659-668.

Zhang, G., Cowled, C., Shi, Z., Huang, Z., Bishop-Lilly, K.A., Fang, X., Wynne, J.W., Xiong, Z., Baker, M.L., Zhao, W., et al. (2013). Comparative analysis of bat genomes provides insight into the evolution of flight and immunity. Science 339, 456-460.

Zhou, H., Chen, X., Hu, T., Li, J., Song, H., Liu, Y., Wang, P., Liu, D., Yang, J., Holmes, E.C., et al. (2020a). A novel bat coronavirus closely related to SARS-CoV-2 contains natural insertions at the S1/S2 cleavage site of the spike protein. Curr Biol 30, 2196-2203.e3.

Zhou, P., Tachedjian, M., Wynne, J.W., Boyd, V., Cui, J., Smith, I., Cowled, C., Ng, J.H.J., Mok, L., Michalski, W.P., et al. (2016). Contraction of the type I IFN locus and unusual constitutive expression of $I F N-\alpha$ in bats. Proc Natl Acad Sci USA 113, 2696-2701.
Zhou, P., Yang, X.L., Wang, X.G., Hu, B., Zhang, L., Zhang, W., Si, H.R., Zhu, Y., Li, B., Huang, C.L., et al. (2020b). A pneumonia outbreak associated with a new coronavirus of probable bat origin. Nature 579, 270-273.

Zuo, T., Liu, Q., Zhang, F., Lui, G.C.Y., Tso, E.Y., Yeoh, Y.K., Chen, Z., Boon, S.S., Chan, F.K., Chan, P.K., et al. (2020a). Depicting SARSCoV-2 faecal viral activity in association with gut microbiota composition in patients with COVID-19. Gut gutjnl-2020-322294.

Zuo, T., Zhan, H., Zhang, F., Liu, Q., Tso, E.Y.K., Lui, G.C.Y., Chen, N., Li, A., Lu, W., Chan, F.K.L., et al. (2020b). Alterations in fecal fungal microbiome of patients with COVID-19 during time of hospitalization until discharge. Gastroenterology, doi: 10.1053/j.gastro.2020.1006.1048.

Zuo, T., Zhang, F., Lui, G.C.Y., Yeoh, Y.K., Li, A.Y.L., Zhan, H., Wan, Y., Chung, A.C.K., Cheung, C.P., Chen, N., et al. (2020c). Alterations in gut microbiota of patients with COVID-19 during time of hospitalization. Gastroenterology 159, 944-955.e8. 